\title{
First Report of Chlamydiaceae Seroprevalence in Tibetan Pigs in Tibet, China
}

\author{
Nian-Zhang Zhang,, Dong-Hui Zhou, Xin-Chun Shi,, ${ }^{1,2}$ Alasdair J. Nisbet, ${ }^{3}$ Si-Yang Huang, \\ Danba Ciren, ${ }^{4}$ Song-Ming Wu, and Xing-Quan Zhu ${ }^{1,5,6}$
}

\begin{abstract}
The seroprevalence of Chlamydiaceae infection in Tibetan pigs in Tibet, China, was examined by indirect hemagglutination assay (IHA), between April, 2010, and December, 2010. A total of 71 of 427 serum samples (16.63\%, 95\% confidence interval [CI] 15.31-17.95] were positive for Chlamydiaceae antibodies. Forty Chlamydiaceae seropositives from 232 samples were recorded in sera from Nyingchi (17.24\%, 95\% CI 15.40-19.08) and 31 positives were recorded in 195 serum samples from Mainling (15.90\%, 95\% CI 14.02-17.78). The investigation showed that the prevalence in female animals was $17.61 \%$ (95\% CI 15.22-20.00), and in male animals it was $12.72 \%$ (95\% CI 11.07-14.37). The prevalence ranged from $0 \%$ to $20.61 \%$ (95\% CI 17.81-23.48) among different age groups, with a higher prevalence in growing pigs $(p<0.01)$. The results indicated that Chlamydiaceae infection was widespread in Tibetan pigs in Tibet, China, which is of public health concern in this region of the world. To our knowledge, this is the first report of Chlamydiaceae seroprevalence in Tibetan pigs in Tibet, China.
\end{abstract}

Key Words: Chlamydiaceae—Seroprevalence-Tibetan pigs-Tibet—Indirect hemagglutination assay.

\section{Introduction}

C HLAMYDIACEAE, OBLIGATE INTRACELLULAR BACTERIA, are the etiological agents of chlamydiosis and are responsible for a broad spectrum of diseases in animals and humans (Raso et al. 2010, Rohde et al. 2010, Schautteet and Vanrompay 2011). Four Chlamydiaceae species have been isolated from pigs: Chlamydia suis, Chlamydophila psittaci, Chlamydophila abortus, and Chlamydophila pecorum (Pantchev et al. 2010, Rohde et al. 2010, Schautteet and Vanrompay 2011), and infections with these agents are usually associated with pneumonia (Reinhold et al. 2008), severe dyspnea (Sachse et al. 2004), conjunctivitis (Becker et al. 2007), arthritis, and necrotizing enteritis in pigs. In addition, numerous reproductive problems such as vaginitis, endometritis, returning to estrus, abortion, mummification, delivery of weak piglets, and increased neonatal mortality in sows (Schautteet and Vanrompay 2011), as well as orchitis, epididymitis, and vesiculitis in boars (Camenisch et al. 2004, Schautteet and Vanrompay 2011), can be caused by infection with members of the Chlamydiaceae.

Chlamydiaceae infections in pigs have been reported worldwide (Eggemann et al. 2000, Vanrompay et al. 2004, Wang et al. 2006, Zhou et al. 2008, Pantchev et al. 2010, Xu et al. 2010); however, little is known about Chlamydiaceae prevalence in Tibetan pigs in Tibet, China. The Tibetan pig is a Chinese native pig breed distributed in the southeastern Tibet Plateau and the surrounding areas. During longterm free-range production, Tibetan pigs have evolved adaptions to low temperature and poor food quality (Xin et al. 2011) and, physically possess powerful musculature, with coarse black hair covering the whole body (Zheng 1998). The animal's meat is a sought-after delicacy with a tender texture and high nutritional value. Thus, Tibetan pigs have become an important source of income for $\mathrm{Ti}$ betans. Tibetan pigs may also act as a potential risk in the

\footnotetext{
${ }^{1}$ State Key Laboratory of Veterinary Etiological Biology, Lanzhou Veterinary Research Institute, Chinese Academy of Agricultural Sciences, Lanzhou, Gansu Province, PR China.

${ }^{2}$ College of Veterinary Medicine, Northwest A \& F University, Yangling, Shaanxi Province, PR China.

${ }^{3}$ Parasitology Division, Moredun Research Institute, Pentlands Science Park, Midlothian, Scotland, United Kingdom.

${ }^{4}$ College of Animal Science, Tibet College of Agriculture and Animal Husbandry, Nyingchi, Tibet Autonomous Region, PR China.

${ }^{5}$ College of Animal Science and Technology, Yunnan Agricultural University, Kunming, Yunnan Province, PR China.

${ }^{6}$ College of Animal Science and Veterinary Medicine, Heilongiiang Bayi Agricultural University, Daqing, Heilongjiang Province, PR China.
} 
Table 1. Prevalence of Antibodes to Chlamydiaceae in Tibetan Pigs of Different Genders and Geographical Locations in Tibet, China, Using Indirect Hemagglutination Assay

\begin{tabular}{lccccc}
\hline Gender & No. examined & $\begin{array}{c}\text { Geographical location } \\
\text { Mainling }\end{array}$ & Nyingchi & No. positive & Prevalence \% (95\% CI) \\
\hline Male & 173 & 12 & 10 & 22 & $12.72(11.07-14.37)$ \\
Female & 142 & 10 & 15 & 25 & $17.61(15.22-20.00)$ \\
Unknown & 112 & 9 & 15 & 24 & $21.43(18.31-24.55)$ \\
Total & 427 & 31 & 40 & 71 & $16.63(15.31-17.95)$ \\
\hline
\end{tabular}

spread of infectious disease. In this context, the objective of the present study was to investigate using a serological approach whether Chlamydiaceae are prevalent in Tibetan pigs in Tibet, China.

\section{Materials and Methods}

\section{Investigation sites}

The present study was carried out in Nyingchi and Mainling counties of the Nyingchi Prefecture, southeastern Tibet. As described by Wu et al. (2012), the average elevation of the surveyed area is more than 3000 meters above sea level, covering about $120,000 \mathrm{~km}^{2}$. This region is influenced by the warm current from the Pacific and Indian Ocean, and has a humid tropical climate.

\section{Serum samples}

A total of 427 Tibetan pig blood samples including 232 from Nyingchi county and 195 from Mainling county were collected by veterinary practitioners between April, 2010, and December, 2010. The samples were centrifuged at $3000 \times g$ for $5 \mathrm{~min}$. Then, the serum was removed and stored at $-20^{\circ} \mathrm{C}$ until further analysis.

\section{Serological tests}

The commercially available indirect hemagglutination assay (IHA) kit (Lanzhou Veterinary Research Institute, Chinese Academy of Agriculture Sciences) was used to examine antibodies to Chlamydiaceae. The detection procedures were carried out as previously reported (Xu et al. 2010). Samples that reacted at dilutions of 1:16 or higher were considered positive for Chlamydiaceae antibodies. Positive results from dilutions of between 1:4 and 1:16 were considered "suspect" and were retested.

\section{Statistical analysis}

Differences in the prevalence of Chlamydiaceae in Tibetan pigs from different geographical locations and age groups were analyzed with a chi-squared test using SPSS for Windows (release 18.0 standard version, SPSS Inc., Chicago, IL). All tests were 2 -sided, and values of $p<0.05$ were considered as statistically significant.

\section{Results and Discussion}

Seventy-one out of 427 serum samples $(16.63 \%$, 95\% confidence interval [CI] 15.31-17.95) were seropositive for Chlamydiaceae by IHA. Antibody titers were 1:256 in 2, 1:128 in 2, 1:64 in 5, 1:32 in 10, and 1:16 in 52 in the seropositive Tibetan pigs. Of the total of 71 seropositive results, 40 (of 232) samples came from Nyingchi $(17.24 \%, 95 \%$ CI 15.40-19.08) and 31 (of 195) originated from Mainling (15.90\%, 95\% CI 14.02-17.78) (Table 1), although the difference in seroprevalnce between the 2 counties was not statistically significant $(p>0.05)$. As shown in Table 1, the investigation revealed that the prevalences in female and male animals were $17.61 \%$ (95\% CI 15.2220.00) and $12.72 \%$ (95\% CI 11.07-14.37), respectively. Among these positive Tibetan pigs, seroprevalence varied in different age groups, ranging from 0 to $20.61 \%$ (95\% CI $17.81-23.48$ ) (Table 2).

Surveys of Chlamydiaceae infections in pigs have been performed in many countries (Eggemann et al. 2000, Vanrompay et al. 2004, Pantchev et al. 2010) and some provinces in China (Suo et al. 2005, Luo et al. 2006, Wang et al. 2006, Xian et al. 2007, Zhou et al. 2008, Xu et al. 2010), but little is known about infection with Chlamydiaceae in Tibetan pigs, which are one of the main sources of food and economic activity in Tibet. In this study, the overall seropositivity for Chlamydiaceae exposure was 16.63\% (95\% CI 15.31-17.95) in Tibetan pigs, which was lower than that of the intensively raised pigs

Table 2. Antibody Titers to Chlamydiaceae Infection in Tibetan Pigs in Tibet, China, by Indirect Hemagglutination Assay

\begin{tabular}{|c|c|c|c|c|c|c|c|c|c|}
\hline \multirow[b]{2}{*}{ Types of pig } & \multirow[b]{2}{*}{ No. examined } & \multicolumn{6}{|c|}{ IHA titers } & \multirow[b]{2}{*}{ No. positive } & \multirow[b]{2}{*}{ Prevalence \% (95\% CI) } \\
\hline & & $\leq 1: 4$ & 1:16 & $1: 32$ & $1: 64$ & $1: 128$ & $1: 256$ & & \\
\hline Breeding boar & 4 & 4 & 0 & 0 & 0 & 0 & 0 & 0 & 0 \\
\hline Breeding sow & 5 & 5 & 0 & 0 & 0 & 0 & 0 & 0 & 0 \\
\hline Slaughter pig & 110 & 91 & 13 & 4 & 0 & 2 & 0 & 19 & 17.27 (14.60-19.94) \\
\hline Fattening pig & 106 & 93 & 12 & 0 & 1 & 0 & 0 & 13 & $12.26(10.22-14.30)$ \\
\hline Growing pig & 131 & 104 & 19 & 3 & 3 & 0 & 2 & 27 & $20.61(17.81-23.48)$ \\
\hline Piglet & 71 & 59 & 8 & 3 & 1 & 0 & 0 & 12 & 16.90 (13.63-20.17) \\
\hline Total & 427 & 357 & 52 & 10 & 5 & 2 & 2 & 71 & 16.63 (15.31-17.95) \\
\hline
\end{tabular}

IHA, indirect hemagglutination assay; CI, confidence interval. 
in other provinces of China (Jin et al. 2005, Li 2005, Suo et al. 2005, Luo et al. 2006, Wang et al. 2006, Xian et al. 2007, Zhou et al. 2008, Xu et al. 2010), but higher than that observed in Yunnan province (10.5\%) in China (Cao and Zhang 2007). The low seropositivity of Chlamydiaceae in Tibetan pigs may be attributed to higher resistance of Tibetan pigs to the disease compared with pigs from intensive farming systems and inbreeding of Tibetan pigs that could decrease the opportunity of transmission through mating with other populations of pigs.

Among different age groups of Tibetan pigs, a higher prevalence $(20.61 \%, 95 \%$ CI $17.81-23.48)$ was detected in growing pigs $(p<0.01)$, whereas no seroprevalence was detected in breeding boars and sows, possibly due to the limited number of samples. In other reports in China, the seropositivity of Chlamydiaceae in breeding boars and sows from intensive farms was usually more than $30 \%$ (Jin et al. 2005, Li 2005, Suo et al. 2005, Luo et al. 2006, Wang et al. 2006, Xian et al. 2007, Zhou et al. 2008, Xu et al. 2010). Further studies of Chlamydiaceae infection in Tibetan pigs should focus on breeding boars and sows.

No statistically significant difference in Chlamydiaceae seroprevalence was observed between female and male Tibetan pigs $(p>0.05)$. However, a tendency for higher prevalence among females was observed. This tendency was also observed in the previous surveys of wild boars in Italy (females, 45.95\%; males, 38.8\%) (Antonietta et al. 2011) and Germany (females, 83.3\%; males, 42.9\%) (Helmut et al. 2004) by microimmunofluorescence (MIF) and PCR. Therefore, further studies should be performed to ascertain whether gender is a crucial factor for Chlamydiaceae infection. In this present study, the Chlamydiaceae seropositivity in the 2 counties did not differ significantly $(p>0.05)$, probably because of the use of similar environmental and free-range systems.

Many wild birds (Kaleta and Taday 2003) and ruminants (Cubero-Pablo et al. 2000) can also carry some species of Chlamydiaceae. Cross-species transmission is widespread in chlamydial circulation (Lemus et al. 2010). Whether Tibetan pigs acquired Chlamydiaceae through wild animals interacting with domestic animal husbandry practices in this region could not be assessed based on the present data. Further investigations should study the transmission of Chlamydiaceae in Tibetan pigs, other animals, and humans in Tibet.

In conclusion, the present study demonstrated that there was a $16.63 \%(95 \%$ CI 15.31-17.95) seroprevalence of Chlamydiaceae infection in Tibetan pigs in Tibet, China, which provided baseline data for the effective prevention and control of chlamydiosis in Tibetan pigs in this unique region of the world. To our knowledge, the present report is the first to document the seroprevalence of Chlamydiaceae infection in Tibetan pigs in China.

\section{Acknowledgments}

Project support was provided, in part, by the Science Fund for Creative Research Groups of Gansu Province (Grant No. 1210RJIA006), the Open Funds of the State Key Laboratory of Veterinary Etiological Biology, Lanzhou Veterinary Research Institute, Chinese Academy of Agricultural Sciences (grant nos. SKLVEB2011KFKT004，SKLVEB2011KFKT011，SKLVEB2010 KFKT009, and SKLVEB2011KFKT010), and the Yunnan Pro- vincial Program for Introducing High-level Scientists (grant no. 2009CI125).

\section{Author Disclosure Statement}

No completing financial interests exist. The findings and conclusions in this report are those of the authors and do not necessarily represent the views of the funding agencies.

\section{References}

Antonietta DF, Manuela D, Federico M, Maria R, et al. Seroepidemiologic survey for Chlamydia suis in wild boar (Sus scrofa) populations in Italy. J Wildlife Dis 2011; 47:709-712.

Becker A, Lutz-Wohlgroth L, Brugnera E, Lu ZH, et al. Intensively kept pigs pre-disposed to chlamydial associated conjunctivitis. J Vet Med A 2007; 54:307-313.

Camenisch U, Lu ZH, Vaughan L, Corboz L, et al. Diagnostic investigation into the role of Chlamydiae in cases of increased rates of return to oestrus in pigs. Vet Rec 2004; 155:593-596.

Cao XP, Zhang YF. Serological survey of porcine chlamydiosis in Chuxiong city of Yunnan province. Chin Anim Husbandry Vet Med 2007; 34:110-111 [in Chinese].

Cubero-Pablo MJ, Plaza M, Perez L, Gonzalez M, et al. Seroepidemiology of chlamydial infections of wild ruminants in Spain. J Wildlife Dis 2000; 36:35-47.

Eggemann G, Wendt M, Hoelzle LE, Jäger C, et al. Prevalence of Chlamydia infections in breeding sows and their importance in reproductive failure. Deutsches Tierarztl Wochenschr 2000; 107:3-10.

Helmut H, Angela B, Falk M, Konrad S. Occurrence of Chlamydiaceae spp. in a wild boar (Sus scrofa L.) population in Thuringia (Germany). Vet Microbiol 2004; 103:121-126.

Jin AH, Gu BL, Wei XY, Fang GM. Serological survey of porcine chlamydiosis in Shanghai and around areas. Shanghai J Anim Husbandry Vet Med 2005; 6:23 [in Chinese].

Kaleta EF, Taday EM. Avian host range of Chlamydophila spp. based on isolation, antigen detection and serology. Avian Pathol 2003; 32:435-461.

Lemus JA, Fargallo JA, Vergara P, Parejo D, et al. Natural cross chlamydial infection between livestock and free-living bird species. PLOS One 2010; 5:e13512.

Li ML. Serological investigation of chlamydiosis in pigs from partial intensive farms in Hunan province. Hunan J Anim Sci Vet Med 2005; 6:36-37 [in Chinese].

Luo J, Yang XY, Dai AL, Li XH. Serological investigation of chlamydiosis in sow pigs from intensive farms. Fujian J Anim Husbandry Vet Med 2006; 28:4-5 [in Chinese].

Pantchev A, Sting R, Bauerfeind R, Tyczka J, et al. Detection of all Chlamydophila and Chlamydia spp. of veterinary interest using species-specific real-time PCR assays. Comp Immunol Microb 2010; 33:473-484.

Raso TF, Carrasco AOT, Silva JCR., Marvulo MFV, et al. Seroprevalence of antibodies to Chlamydophila psittaci in zoo workers in Brazil. Zoonoses Public Health 2010; 57:411-416.

Reinhold P, Kirschvink N, Theegarten D, Berndt A. An experimentally induced Chlamydia suis infection in pigs results in severe lung function disorders and pulmonary inflammation. Vet Rec 2008; 39:35.

Rohde G, Straube E, Essig A, Reinhold P, et al. Chlamydial zoonoses. Deutsches Ärzteblatt Inter 2010; 107:174-180.

Sachse K, Grossmann E, Berndt A, Schutt C, et al. Respiratory chlamydial infection based on experimental aerosol challenge of pigs with Chlamydia suis. Comp Immunol Microb 2004; $27: 7-23$ 
Schautteet K, Vanrompay D. Chlamydiaceae infections in pig. Vet Rec 2011; 42:29.

Suo XF, Wang DL, Zhang SH, Wang JX, et al. Serological investigation of porcine chlamydiosis in pig from intensive farms in Hainan province. Swine Prod 2005; 3:31-32 [in Chinese].

Vanrompay D, Geens T, Desplanques A, Hoang TQ, et al. Immunoblotting, ELISA and culture evidence for Chlamydiaceae in sows on 258 Belgian farms. Vet Microbiol 2004; 99:59-66.

Wang JH, Chen L, Ren XY, Wu Q, et al. Serological investigation of porcine chlamydiosis of the parts of several regions in Henan province. Progress Vet Med 2006; 27:88-89 [in Chinese].

Wu SM, Ciren D, Huang SY, Xu MJ, et al. First report of Toxoplasma gondii prevalence in Tibetan Pigs in Tibet, China. Vector Borne Zoonotic Dis 2012; 12:654-656.

Xian QZ, Cai MZ, Wang SM, Huang LZ. Serological investigation and control of chlamydiosis in pig from small farms in partial areas of Guangdong and Guangxi province. Chin J Anim Husbandry Vet Med 2007; 1:20-21 [in Chinese].

Xin SP, Shi D, Zhang J. Research of protection, development and utilization of genetic resources of Tibetan pig in Tibet Au- tonomous Region. Chin Anim Husbandry Bull 2011; 4:49-51 [in Chinese].

Xu MJ, He Y, Liang R, Zhou DH, et al. Seroprevalence of Chlamydia infection in pigs from intensive farms in Southern China. J Anim Vet Adv 2010; 9:1143-1145.

Zheng Z. A typical high plateau pig breed-Tibetan pig. Jilin J Anim Vet Med 1998; 11:18 [in Chinese].

Zhou P, Lin ZY, Wang TF, Luo CQ, et al. Investigation on seral epidemiolog of porcine chlamydiosis in several regions of Longyan city. Progress Vet Med 2008; 29:30-35 [in Chinese].

Address correspondence to: Xing-Quan Zhu State Key Laboratory of Veterinary Etiological Biology

Lanzhou Veterinary Research Institute Chinese Academy of Agricultural Sciences Lanzhou, Gansu Province 730046 The People's Republic of China

E-mail: xingquanzhu1@hotmail.com 